\title{
Die demokratische Grenze output-orientierter Legitimation
}

\author{
Armin Schäfer*
}

In jeder Demokratie wird eine Grenze gezogen: Was muss politisch, was kann bürokratisch entschieden werden? Alle Demokratien definieren Bereiche, in denen das Mehrheitsprinzip nicht gilt, sondern Experten unabhängig entscheiden. Diese Experten werden häufig ernannt und nicht gewählt. Die Delegation von Aufgaben an nicht-politische Akteure soll nicht nur Entscheidungen effizienter, sondern auch deren Umsetzung effektiver machen und so die Leistungsfähigkeit des politischen Systems verbessern. Doch wo genau die Grenze zwischen beiden Bereichen gezogen werden soll, ist schon im Nationalstaat umstritten. Besondere Brisanz gewinnt diese Frage in der Europäischen Union. Denn es ist unklar, ob eine Politisierung der EU-Entscheidungen positive Effekte hätte oder ob die Auseinandersetzung um Policies in Konflikte über die Integration umschlüge. ${ }^{1}$ Diese Debatte betrifft die Frage wie die Demokratie in der EU gestärkt werden kann, ohne die Integration zu gefährden. Einige Autoren bestreiten jeglichen Bedarf an zusätzlicher Input-Legitimation. Sie sehen die Hauptaufgabe der EU darin, Problemlösungen anzubieten wo dies den Mitgliedstaaten nicht gelingt. Nicht-majoritäre Institutionen sind aus dieser Sicht gerechtfertigt, weil sie die Output-Legitimität erhöhen und auf diese Weise die Akzeptanz der Politik steigern. Die im Vergleich zum Nationalstaat höhere Handlungsfähigkeit der EU wird dadurch erreicht, dass die Entscheidungsgremien ,evade the control and constraint of popular democracy and accountability."2 Eine Politisierung der EU-Institutionen hätte zur Folge, dass sie diese Funktion nicht länger erfüllen könnten.

Dieser Aufsatz greift die Debatte um das Demokratiedefizit der EU auf, indem er den Delegationsansatz und dessen Annahmen darstellt. Entscheidungen, die nicht unmittelbar aus dem demokratischen Prozess hervorgehen, werden mit dem Verweis auf die Problemlösungsfähigkeit des politischen Systems begründet. Die EU könne dort auf Input-Legitimität verzichten, wo ihre Entscheidungen Pareto-Verbesserungen ${ }^{3}$ ermöglichen, die den Mitgliedstaaten sonst verwährt blieben. In der EU bestehe kein Demokratiedefizit, weil sich die Grenzziehung zwischen Politik und Regulierungsbehörden nicht prinzipiell von der in den Mitgliedstaaten unterscheide und technokratische Entscheidungen nur in politisch unstrittigen Bereichen stattfänden. Dagegen wendet dieser Aufsatz ein, dass die Übertragung von Aufgaben an schwach legitimierte Akteure nur dann demokratisch unbedenklich ist, wenn Pareto-Verbesserungen allokativ neutral sind, keine indirekten Verteilungseffekte zwischen Politikfeldern auftreten und Entscheidungen reversibel sind. Werden diese Bedingungen unvollständig erfüllt, erhöht sich die Anforderung an die demokratische Qualität der Verfah-

* Dr. Armin Schäfer, Max-Planck-Institut für Gesellschaftsforschung, Köln.

Für wertvolle Hinweise danke ich Michael Blauberger, Holger Döring, Martin Höpner, Wendelmoet van den Nouland, Britta Rehder, Ulrich Sedelmeier, Wolfgang Streeck, Christine Trampusch, Cornelia Woll und den anonymen Gutachtern.

1 Siehe hierzu die Beiträge von Simon Hix und Stefano Bartolini: Politics: The Right or Wrong Sort of Medicine for the EU?, Notre Europe Policy Paper Nr. 19/2006,

www.notre-europe.asso.fr/IMG/pdf/Policypaper19-en.pdf (letzter Zugriff: 12.06.2006).

2 Peter Mair: Popular Democracy and the European Union Polity, in: European Governance Papers (EUROGOV) C-05-03/2005, S. 22.

3 Das Pareto-Optimum bezeichnet einen Zustand, in dem kein Individuum besser gestellt werden kann, ohne ein anderes dadurch schlechter zu stellen. 
ren. Beschlüsse, die Verlierer produzieren, können deren Zustimmung nur erhoffen, wenn sie das Ergebnis eines transparenten und fairen Verfahrens sind. Zudem müssen die Unterlegenen erwarten können, dass sie in der Lage sein werden, eine andere Politik durchzusetzen, falls sie die Mehrheit erringen. Politische Entscheidungen gelten als legitim, wenn auch diejenigen mit abweichenden Interessen und Präferenzen bereit sind, ihnen ohne Zwang Folge zu leisten. Je stärker EU-Entscheidungen innenpolitisch umkämpfte Politikfelder betreffen, desto weniger lässt sich fehlende Input-Legitimation durch eine verbesserte Performanz des politischen Systems beheben, denn was als ,Problemlösung' gilt wird selbst Gegenstand politischer Kontroversen.

Der Aufbau des Aufsatzes ist wie folgt: Im nächsten Abschnitt werden die Grundzüge der Principal-Agent-Theorie anhand der Literatur zu unabhängigen Notenbanken und zum amerikanischen Kongress dargestellt. Der darauf folgende Abschnitt erläutert, wie dieser Theorietradition entliehene Argumente genutzt werden, die Kritik am Demokratiedefizit der EU zurückzuweisen. Indem die Aufgaben der EU-Institutionen denen von Bürokratien gleichgestellt werden, kann die Frage nach demokratischer Repräsentation vernachlässigt werden. Die bekanntesten Vertreter der ,Gegenthese` zum Demokratiedefizit sind Majone und Moravcsik. Beide verweisen auf die Logik der Delegation, um nicht-majoritäre Entscheidungen der EU-Institutionen zu begründen. Eine kritische Prüfung ihrer Argumente zeigt, dass die Voraussetzungen, von denen die Legitimität delegierter Entscheidungen abhängt, in vielen Politikfeldern der Europäischen Union nicht erfüllt sind. Die Zwecke sind nicht unkontrovers, sondern Gegenstand politischer Auseinandersetzungen.

Die Unbedenklichkeit entpolitisierter Entscheidungen hängt davon ab, dass die beauftragten Akteure politisch unstrittige Problemlösungen implementieren. Während die Entscheidungen der EU längst mehr als verteilungsneutrale Pareto-Verbesserungen sind, fallen die angewandten Verfahren hinter den Maßstab demokratischer Repräsentation zurück. Deshalb wächst mit dem Souveränitätstransfer auf die EU auch die Anforderung an die gewählten Verfahren. Im politischen System der EU mangelt es jedoch an der Artikulation von Kritik und Alternativen. Weder die Wahlen zum Europäischen Parlament noch die Ernennung der Kommission erlauben die Auswahl konkurrierender Konzepte für die EU-Politik. Demokratischer Streit zwischen Regierung und Opposition findet kaum statt. Noch dazu haben nicht alle Wählerstimmen die gleiche Chance, in intergouvernementalen EU-Gremien repräsentiert zu werden, weil Parteien am Rand des politischen Spektrums mit geringerer Wahrscheinlichkeit in Regierungen vertreten sind. Da gleichzeitig die Kontrollfähigkeit nationaler Parlamente abnimmt, verlieren Stimmen für die Oppositionsparteien an Gewicht. Damit gefährdet das politische System der EU den Grundsatz ,horizontaler Gleichheit“ zwischen den Wählern. ${ }^{4}$ Das Demokratiedefizit der EU, so lautet der Befund der folgenden Diskussion, besteht im Kern in der Entpolitisierung der EU-Politik.

\section{Delegation, Effizienz und Kontrolle: das Principal-Agent-Problem}

Die Delegation von Aufgaben bedeutet, dass ein Akteur (agent oder Stellvertreter) im Auftrag eines anderen Akteurs (principal oder Auftraggeber) handelt. ${ }^{5}$ Ein Delegationsverhältnis enthält die temporäre Verlagerung von Entscheidungsbefugnissen auf den Stellvertreter. Aufgaben werden häufig dort übertragen, wo die Auftraggeber selbst nicht zu

4 Christopher Lord: Democracy and Democratization in the European Union, in: Simon Bromley (Hrsg.): Governing the European Union, London 2001, S.165-190, hier S. 167.

5 Stephen A. Ross: The Economic Theory of Agency: The Principal's Problem, in: American Economic Review 2/1973, S. 134-139, hier S. 134. 
effizienten Entscheidungen fähig sind. Das Grundproblem liegt dabei in der Informationsasymmetrie zwischen den Akteuren. ${ }^{6}$ Der Stellvertreter erwirbt durch seine Tätigkeit einen Informationsvorsprung, den der Auftraggeber nur mit hohem Aufwand überwinden kann. Vor allem zwei Literaturstränge haben das Delegationskonzept zur Analyse politikwissenschaftlicher Fragestellungen angewandt: Zum einen Studien zur Unabhängigkeit von Zentralbanken und zum anderen Arbeiten zur Funktionsweise des amerikanischen Kongresses. Beide Stränge werden im Folgenden skizziert.

Das Argument für eine politisch unabhängige Zentralbank besteht in der erwarteten Diskrepanz zwischen einer langfristig angemessenen Geldpolitik und den kurzfristigen, wahlstrategischen Bedürfnissen der Regierung, die der „politische Konjunkturzyklus“ beschreibt. ${ }^{7}$ Selbst wenn eine Regierung für Geldwertstabilität eintritt, bestehen in Zeiten leerer Kassen Anreize, von diesem Ziel abzuweichen. Da Steuererhöhungen unpopulär sind, kann eine weisungsgebundene Zentralbank stattdessen zur Bereitstellung von Notenbankkrediten aufgefordert werden, um steigende Staatsausgaben zu bedienen. Die Politik ist der Versuchung ausgesetzt, entgegen vorheriger Ankündigungen eine expansive Geldpolitik zur Stimulierung des Wachstums zu verfolgen. ${ }^{8}$ Allerdings antizipieren und berücksichtigen die anderen Wirtschaftsakteure diese Absicht in ihrem Verhalten. So durchschauen die Gewerkschaften die Geldwertillusion und kämpfen in Tarifverhandlungen für eine Inflationsprämie, die den Anstieg der Reallöhne gewährleisten soll. Die angekündigte stabilitätsorientierte Politik leidet unter einem inhärenten Glaubwürdigkeitsproblem, mit der Folge, dass die Inflation - ohne positive realwirtschaftliche Effekte - über dem gewünschten Maß liegt. Nur über die Eingrenzung des diskretionären Spielraums politischer Entscheidungsträger kann Glaubwürdigkeit erzielt werden. Die Unabhängigkeit der Zentralbank dient aus dieser Sicht der Abwehr eines opportunistischen oder zeitinkonsistenten Verhaltens der Politik. ${ }^{9}$

Für die Geldpolitik liegt die Begründung der Delegation von Aufgaben an unabhängige agents in der Erwartung, dass deren Präferenzen von denen der principals abweichen. Zentralbanken sollen sich politische Anliegen nicht zu Eigen machen, sondern ihre Entscheidungen dem Ziel stabiler Preise unterordnen. Entgegen des sonst häufig formulierten Problems delegierter Befugnisse steht im besonderen Fall der Zentralbankunabhängigkeit die Kontrolle der agents nicht im Vordergrund. ${ }^{10}$

Ganz anders wird Delegation im Fall des amerikanischen Kongresses diskutiert. Hier geht es um die Frage, wie bürokratische Eigenmächtigkeit wirkungsvoll eingegrenzt werden kann. Delegation enthält immer Opportunitätskosten, die gegen den erwarteten Nutzen abgewogen werden müssen. Der Kongress misst die Vorteile von Delegation an den notwendig damit verbundenen, jedoch unerwünschten Kosten. ${ }^{11}$ Fiorina argumentiert, dass der Kongress Entscheidungen auf die Bürokratie verschiebt, wenn die Kosten konzentriert sind und der Nutzen diffus bleibt, um nicht für unpopuläre Entscheidungen verantwortlich ge-

6 Roderick D. Kiewiet/Mathew D. McCubbins: The Logic of Delegation. Congressional Parties and the Appropriations Process, Chicago/London 1991, S. 25.

7 Alesina Alberto/Gerald D. Cohen/Nouriel Roubini: Electoral Business Cycle in Industrial Democracies, in: European Journal of Political Economy 1/1993, S. 1-23.

8 Bernhard William/J. Lawrence Broz/William Roberts Clark: The Political Economy of Monetary Institutions, in: International Organization 4/2002, 693-723, hier S. 693 und 705.

9 Rolf Caesar: Die Unabhängigkeit der Notenbank im demokratischen Staat. Argumente und Gegenargumente, in: Zeitschrift für Politik 4/1980, S. 347-377, hier S. 351-352.

10 Gary J. Miller: The Political Evolution of Principal-Agent Models, in: Annual Review of Political Science 2005, S. 203-225, hier S. 218.

11 Mathew D. McCubbins/Roger G. Noll/Barry R. Weingast: Administrative Procedures as Instruments of Political Control, in: Journal of Law, Economics, \& Organization 2/1987, S. 243-277. 
macht zu werden, jedoch im gegenteiligen Fall selbst entscheidet. ${ }^{12}$ Epstein und O`Halloran entwickeln eine Theorie der Delegation anhand der Charakteristik der Policy sowie des Verhältnisses vom Kongress zur Exekutive (Präsidentialbürokratie) und zu den parlamentarischen Ausschüssen. Ist der Informationsbedarf (Expertise) eines Themas hoch, der Verteilungseffekt aber gering, delegiert der Kongress die Entscheidung oder Bearbeitung eher an spezialisierte Ausschüsse oder die Bürokratie als im umgekehrten Fall. Zudem üben die Mehrheitsverhältnisse Einfluss auf die Delegationsentscheidung aus. Stimmt die parteipolitische Mehrheit des Kongresses mit der Parteizugehörigkeit des Präsidenten überein (unified government) ist Delegation an die Bürokratie wahrscheinlicher als bei adversen Mehrheiten (divided government). Die Autoren verfechten eine Theorie rationaler Delegation. Aufgaben werden delegiert, um den politischen Nutzen für die Auftraggeber zu maximieren. ${ }^{13}$

Delegation soll die Handlungsfähigkeit der Politik erhöhen, birgt aber auch Risiken. Delegierte Entscheidungen sind der demokratischen Kontrolle durch gewählte Repräsentanten partiell entzogen. Entscheiden agents von der Öffentlichkeit abgeschottet, steigt die Wahrscheinlichkeit, dass sie von Partikularinteressen vereinnahmt werden (agency capture). In diesem Fall sorgt nicht der Eigensinn der Stellvertreter, sondern der Einfluss organisierter Interessen für ein vom Auftraggeber abweichendes Verhalten. In der Literatur werden zwei Varianten der Agency-Kontrolle unterschieden: ${ }^{14}$ Zum einen die Ex-ante-Begrenzung von Reichweite, Instrumenten und Entscheidungsverfahren. Durch die Festlegung der Befugnisse soll ein mit den Wünschen des Kongresses übereinstimmendes Verhalten der Bürokratie gewährleistet werden. Zum anderen besteht die Möglichkeit nachträglicher Kontrolle. Abweichendes Verhalten kann durch Sanktionen bestraft, eine erfolgreiche Bewältigung der Aufgabe belohnt werden. Auch hier besteht das Problem, dass Ressourcen zur Kontrolle aufgewandt werden müssen und ein Informationsgefälle zwischen den Akteuren besteht.

Die Literatur bietet also zwei Begründungen für die Delegation von Aufgaben: Glaubwürdigkeit und effiziente Entscheidungen. Im ersten Fall muss der agent einen vom principal unabhängigen, politisch nicht manipulierbaren Willen haben. Im zweiten Fall entscheiden die principals unter Abwägung von Kosten und Nutzen darüber, ob und in welchem Ausmaß sie Aufgaben delegieren. Effiziente Entscheidungen werden ins Verhältnis zu den Kosten der agency drift gesetzt. Ex-ante-Begrenzung der Befugnisse und Ex-post-Überwachung sollen ein hohes Maß an Übereinstimmung zwischen Auftraggeber und Stellvertreter gewährleisten.

\section{Das Demokratiedefizit der EU}

In der Debatte um das Demokratiedefizit der Europäischen Union werden beide Motive für Delegation aufgegriffen - effizientere Entscheidungsverfahren und eine erhöhte Glaubwürdigkeit der Politik. Mit dem Verweis auf die positiven Effekte unpolitischer Entscheidungen für die Bürger wird hinterfragt, ob in der EU ein Demokratiedefizit besteht. Trotz der Unterschiede in ihrer Argumentation lassen sich vor allem Andrew Moravcsik und Giandomenico Majone dieser Sichtweise zuordnen. Im Kern begründen sie den Kompetenztransfer an nicht-majoritäre Akteure über die verbesserte Leistungsfähigkeit des politischen Systems. Die EU soll das Politikversagen der Mitgliedstaaten ausgleichen und Probleme lösen,

12 Morris Fiorina: Legislative Choice of Regulatory Forms: Legal Process or Administrative Process?, in: Public Choice 1/1982, S. 33-61.

13 David Epstein/Sharyn O'Halloran: Asymmetric Information, Delegation, and the Structure of Policy-making, in: Journal of theoretical politics 1/1999, S. 37-56.

14 Mathew C. McCubbins/Talbot Page: A Theory of Congressional Delegation, in: Mathew D. McCubbins/Terry Sullivan (Hrsg.): Congress: Structure and Policy, Cambridge 1987, S. 409-425, hier S. 411-414. 
die sich im Nationalstaat nicht länger erfolgreich bearbeiten lassen. Die Übertragung von Aufgaben an nicht-gewählte Akteure ist gerechtfertigt, solange ein Wohlfahrtsgewinn erzielt wird. Eine erhöhte Output-Legitimität reduziert aus dieser Sicht die Anforderung an Inputorientierter Legitimation. Die Argumentation der beiden Autoren wird im Folgenden dargestellt, um anschließend eine kritische Diskussion vorzunehmen.

\section{Die Gegenthese: Legitimität durch Leistung}

Moravcsiks Ausgangspunkt ist die Feststellung, dass der autonome Einfluss der EU häufig übertrieben wird. ${ }^{15}$ Die Kompetenzen der Union sind eng definiert, ihre verfügbaren Mittel gering und die Entscheidungen unterliegen hohen Zustimmungshürden, sofern sie überhaupt vom Einstimmigkeitsprinzip abweichen. Das politische System der EU zeichnet sich durch Machtteilung aus, die über das Maß im Nationalstaat hinausgeht. Entscheidungen der EU sind über zwei Kanäle legitimiert. Zum einen indirekt über die nationalen Regierungen, die in den meisten Fällen über ein formales oder informelles Vetorecht verfügen. Zum anderen über das direkt gewählte Europäische Parlament, dessen Stellung in der EU-Gesetzgebung kontinuierlich aufgewertet wurde. Dass das Europäische Parlament in vielem nicht einem nationalen Parlament entspricht, wird durch die Anforderung entschärft, dass Gesetze im Zusammenspiel mit dem Ministerrat verabschiedet werden. Wechselseitig aufeinander bezogen, gewährleisten diese Institutionen demokratische Beschlüsse. Die Angst vor einem unkontrollierten europäischen Superstaat ist aus dieser Perspektive eine Chimäre der britischen Boulevardpresse.

Anknüpfend an die intergouvernementale Lesart der Integration bestreitet Moravcsik den Kontrollverlust der Mitgliedstaaten. Im Zentrum europäischer Politik steht der Ministerrat, in dem die nationalen Regierungen vertreten sind. Der Handlungsspielraum supranationaler Akteure - vor allem der Kommission - ist geringer, als dies landläufig angenommen wird. Zudem vollzieht sich die Delegation von Entscheidungsbefugnissen in der EU nach dem gleichen Muster wie im Nationalstaat:

„The critical point for the study of the EU is this: within the multi-level governance system prevailing in Europe, EU officials (or insulated national representatives) enjoy the greatest autonomy in precisely those areas - central banking, constitutional adjudication, criminal and civil prosecution, technical administration and economic diplomacy - in which many advanced industrial democracies, including most Member States of the EU, insulate themselves from direct political contestation. The apparently 'undemocratic' nature of the EU as a whole is largely a function of this selection effect." 16

Die Begründung für delegierte Entscheidungsbefugnisse liegt im Bemühen, die Problemlösungsfähigkeit der Politik zu erhöhen. Da Verträge unvollständig sind und die Gefahr opportunistischen Verhaltens besteht, müssen Regierungen ihre Vereinbarungen absichern. Eine Möglichkeit wären präzise Verhaltensregeln, deren Befolgung der EuGH überwacht. Doch in Ungewissheit darüber, welche zukünftigen Maßnahmen zur Verwirklichung der vereinbarten Ziele ergriffen werden müssen, erweisen sich ex ante definierte Regeln als untauglich. ${ }^{17}$ Daher verständigen sich die Regierungen auf die Delegation von Entscheidungsbefugnissen in dynamischen Politikfeldern mit hohen Kooperationsgewinnen, wo das Be-

15 Andrew Moravcsik: In Defence of the 'Democratic Deficit': Reassessing Legitimacy in the European Union, in: Journal of Common Market Studies 4/2002, S. 603-624.

16 Ebenda, S. 613, Hervorhebung im Original.

17 Giandomenico Majone: Dilemmas of European Integration. The Ambiguities and Pitfalls of Integration by Stealth, Oxford 2005, S. 72. 
kenntnis zu wirtschaftspolitischen Zielen den Marktteilnehmern glaubwürdig dokumentiert werden muss. ${ }^{18}$ Delegation hilft, die gemeinsam beschlossenen Ziele zu verwirklichen, und verbessert so die Leistungsfähigkeit des politischen Systems. Ein Demokratiedefizit der EU besteht nicht, solange kein unrealistisches Ideal als Bewertungsmaßstab herangezogen wird. ${ }^{19}$

Schließlich bestreitet Moravcsik, dass die EU-Politik wirtschaftliche Belange privilegiert und somit von den Präferenzen der Bevölkerungsmehrheit abweicht, die den Erhalt des Wohlfahrtsstaats wünscht. ${ }^{20}$ Die von ihm als ,sozialdemokratisch“ titulierte These einer Schieflage zugunsten marktschaffender Maßnahmen wird zurückgewiesen, da sich in der Sozial- und Steuerpolitik kein Unterbietungswettbewerb nachweisen ließe. Zudem könne niemand bestreiten, dass die Sozialsysteme in ihrer jetzigen Form nicht aufrecht zu erhalten seien und eines ,neoliberalen“ Korrektivs bedürften. An dieser Stelle wird deutlich, wie politische Überzeugungen in die Argumentation einfließen. Die Europäische Union soll den Mitgliedstaaten zu einer als richtig erkannten Politik verhelfen, die sie innenpolitisch sonst nicht durchsetzen könnten.

Auch für Majone wiegen die Vorteile von Delegation mögliche Nachteile auf. Der Transfer von Kompetenzen ist gerechtfertigt, wo sachgerechte Entscheidungen durch Regulierungsbehörden Marktversagen ausgleichen. Das Ziel der Delegation liegt in der Verwirklichung von Pareto-Verbesserungen. ${ }^{21}$ Aufgrund des geringen Budgets, der Nachfrage nach einheitlichen Regeln und dem Streben der Kommission nach Kompetenzausweitung, hat sich die EU zum ,regulativen Staat“ entwickelt. Ihre Hauptaufgabe liegt in der Vereinheitlichung technischer Regulierung mit dem Ziel, die Funktionsfähigkeit des Gemeinsamen Markts zu verbessern.

Majone benennt beide oben aufgeführten Motive für die Verlagerung von Kompetenzen und unterscheidet zwei Logiken der Delegation. Einerseits verbessert Depolitisierung die Qualität der Entscheidungen, andererseits erhöht sie die Glaubwürdigkeit der Politik. Ist das Ziel die Reduktion von Transaktionskosten leisten die agents Zuarbeit für die principals. Sie stellen Expertise bereit, entwickeln Vorlagen und treffen vorwiegend technische Entscheidungen, die nicht länger einzeln ausgehandelt werden müssen. In diesem Fall ist es wünschenswert, dass die Stellvertreter ihren Auftrag möglichst getreu der Wünsche der Auftraggeber erfüllen. Beispiele sind die Arzneimittelregulierung, Lebensmittelsicherheit oder die Regulierung des Telekommunikationsmarkts. Liegt das vorrangige Ziel von Delegation dagegen in der Glaubwürdigkeit der Politik, darf der agent nicht von den politischen Wünschen der principals abhängig sein. Seine Unabhängigkeit garantiert dauerhaft konsistentes Verhalten. Werden die Entscheidungsbefugnisse vollständig übertragen, spricht Majone von einem Treuhandverhältnis (,fiduciary relation“). ${ }^{22}$

In Abgrenzung zur Kritik am Demokratiedefizit verteidigt Majone unpolitische Entscheidungen. Nicht-majoritäre Institutionen rechtfertigen sich über den Wohlfahrtsgewinn, der mit ihren Entscheidungen einhergeht. ${ }^{23}$ Da Pareto-Verbesserungen per definitionem niemanden schlechter stellen, gibt es keinen Grund für eine Politisierung der Beschlussfassung. Im

18 Andrew Moravcsik: The Choice for Europe. Social Purpose and State Power from Messina to Maastricht, London 1998, S. 73-77.

19 Andrew Moravcsik: Is there a 'Democratic Deficit' in World Politics? A Framework for Analysis, in: Government and Opposition 2/2004, 336-363, hier S. 337.

20 Moravcsik: In Defence of the 'Democratic Deficit', S. 617-619.

21 Giandomenico Majone: The Rise of the Regulatory State in Europe, in: West European Politics 3/1994, S.77101, hier S. 81.

22 Giandomenico Majone: Two Logics of Delegation. Agency and Fiduciary Relations in EU Governance, in: European Union Politics 1/2001, S.103-122, hier S. 105. 
Gegenteil, wenn die Ursache für die Delegation von Aufgaben an unabhängige Behörden in den Nachteilen politischer Entscheidungen liegt, wäre es kontraproduktiv, dem vermeintlichen Demokratiedefizit durch die Aufwertung der Politik zu begegnen. Diesen Trend beobachtet Majone jedoch in der EU. Die politische Kontrolle der Kommission durch nationale Regierungen, die die Kommissare entsenden, und die Aufwertung des Europäischen Parlaments drängen Sachverstand und Neutralität zurück und gefährden jene Glaubwürdigkeit, die durch Delegation erzielt werden soll. Die EU leidet aus seiner Sicht weniger an einem Demokratie- als an einem Glaubwürdigkeitsdefizit: "the progressive politicization and parliamentarization of the Commission raise again the issue of credibility, this time at the European level. A less technocratic, more political Commission may enjoy greater democratic legitimacy, but eventually it will have to face the same commitment problem of all democratic governments." 24

Die Repräsentation unterschiedlicher Interessen ist nicht entscheidend, falls die Ziele nicht umstritten sind. Allerdings ist Majones Argumentation kein Plädoyer für eine unkontrollierte Herrschaft nicht gewählter Akteure. Er fordert stattdessen, die Kompetenzen der EU auf jene Felder zu begrenzen, die unstrittig sind. Für eine redistributive Politik reicht die Legitimationsgrundlage nicht aus, auch weil die Unionsbürger keine belastbare gemeinsame Identität aufweisen. Aus seiner Sicht überdehnt die Europäische Union bereits heute ihre Kompetenzen. ${ }^{25}$

Beide Autoren argumentieren, dass Delegation die Leistungsfähigkeit der Politik verbessert. Sie bestreiten nicht den Mangel an Input-orientierter Legitimation (Herrschaft durch das Volk), verweisen aber auf die legitimierende Wirkung guten Regierens (Herrschaft für das Volk). In der ersten Perspektive geht es darum, politische Entscheidungen möglichst eng an den Willen des Souveräns zu binden. In der zweiten Perspektive steht das Ziel im Vordergrund, die Interessen der Bevölkerung möglichst wirksam zu fördern. ${ }^{26}$ Nicht-majoritäre Entscheidungen beziehen ihre normative Kraft aus Wohlfahrtsgewinnen, die weder durch den Tausch der Marktakteure noch durch nationale Politik erzielt werden. Die Mitgliedstaaten bearbeiten durch die EU Probleme, deren Reichweite sich dem Zugriff nationaler Politik entzieht. Gerade weil wirksame Legitimierungsmechanismen in der Europäischen Union schwieriger als im Nationalstaat umzusetzen sind, gewinnen die Politikergebnisse an Bedeutung. Das Pareto-Kriterium nimmt den Autoren die Beweislast, dass die von ihnen bevorzugten Policies tatsächlich anderen Entscheidungen überlegen sind.

\section{Die demokratische Grenze Output-orientierter Legitimation}

Im Folgenden werden vier Argumente gegen die eben dargestellte Gegenthese „Legitimität durch Leistung" entwickelt. Es wird hinterfragt, ob Kernbereiche der Politik in administrative Entscheidungen umgewandelt werden können. Wo europäische Beschlüsse den Bereich unstrittiger Problemlösung überschreiten, liegt die Herausforderung der EU darin, wirkungsvolle Mechanismen der Repräsentation oder der Partizipation zu finden. In Kürze lauten die Argumente:

- Pareto-Verbesserungen motivieren aus sich heraus nicht notwendig Zustimmung.

23 Allerdings bleibt unklar, auf welcher Ebene sich diese Zugewinne einstellen sollen: auf Ebene der Mitgliedstaaten oder der Bürger?

24 Giandomenico Majone: The Credibility Crisis of Community Regulation, in: Journal of Common Market Studies 2/2000, S. 273-302, hier S. 289.

25 Majone: Dilemmas of European Integration, S. 33 und 36.

26 Fritz W. Scharpf: Legitimationskonzepte jenseits des Nationalstaats, MPIfG Working Paper 6/2004. 
- Der Transfer von Entscheidungskompetenzen ist nur bei einer intakten Repräsentationskette unproblematisch, bei der der Europäische Rat die Wählerpräferenzen widerspiegelt.

- Soll Delegation die Glaubwürdigkeit der Politik erhöhen, ist eine politische Kontrolle der beauftragten Akteure nicht gewollt. In diesem Fall kann die demokratische Legitimität nur aufrechterhalten werden, wenn das Prinzip der Reversibilität eingehalten wird. Ändern sich die politischen Mehrheitsverhältnisse, muss sich dies in Politikänderungen niederschlagen können, da Wahlen sonst ihrer Substanz beraubt werden.

- Schließlich gilt, dass der Souveränitätstransfer keiner Generalbevollmächtigung unzureichend kontrollierbarer Akteure gleichkommen darf. Die Nebenfolgen delegierter Entscheidungen müssen begrenzt sein und dürfen nicht von den Beauftragten selbst definiert werden.

Pareto-Verbesserungen: Formuliert man die Hauptaufgabe nicht-majoritärer Institutionen anhand des Pareto-Kriteriums, wie es delegationstheoretische Argumente nahe legen, erscheint eine zusätzliche Legitimierung der EU unnötig, da alle Betroffenen entweder profitieren oder zumindest nicht schlechter gestellt werden. Doch selbst Pareto-Verbesserungen können Verteilungskonflikte aufwerfen wenn die Wohlfahrtsgewinne ungleich verteilt sind oder die Ausgangssituation nicht als fair anerkannt wird. ${ }^{27}$ Die ökonomische Theorie nimmt an, dass Akteure nicht am relativen Gewinn interessiert sind. Ausschließlich der Vergleich mit dem Status quo entscheidet über Zustimmung oder Ablehnung. Aber wird eine Regierung einem Verhandlungsergebnis zustimmen, von dem ausschließlich andere Staaten profitieren? Natürlich muss nicht jede Einzelentscheidung für alle vorteilhaft sein. Paketlösungen und Ausgleichszahlungen können Verlierer entschädigen. In der EU hat sich zudem die Erwartung herausgebildet, dass heutige Zugeständnisse zukünftig vergolten werden. Doch diese Form , diffuser Reziprozität' hängt von der Fairness des Verfahrens ab. Das gleiche gilt wenn zwar die Gesamtwohlfahrt steigt, aber einzelne gesellschaftliche Gruppen für ihre Verluste entschädigt werden müssen. Wenn Gewinne ungleich verteilt sind, muss ein Verteilungsschlüssel gefunden werden, der diejenigen entschädigt, die sich relativ schlechter stellen. Doch diese Aufteilung lässt sich nicht formal ableiten, denn dies ist eine im Kern politische Entscheidung. Da sie ausschließlich den Gesamtnutzen betrachten, erweisen sich utilitaristische Konzepte als ungeeignet, Fragen der Verteilungsgerechtigkeit befriedigend $\mathrm{zu}$ beantworten. ${ }^{28}$ Ohne substanzielle Übereinkunft gilt das Ergebnis als gerecht, wenn das Verfahren, das es hervorgebracht hat, als fair empfunden wird: ,outcomes are democratically legitimate if and only if they could be the object of a free and reasoned agreement among equals. “29 Damit verschiebt sich der Fokus jedoch von der Effizienz der Verfahren auf die angemessene Repräsentation unterschiedlicher Interessen - und damit auf die Frage, wie Input-orientierte Legitimation gewährleistet werden kann.

Kompetenztransfers: Auf den ersten Blick erscheinen Verteilungsfragen für die Legitimität europäischer Politik dennoch unproblematisch, weil Regierungen sich in Kenntnis des Ausgangspunkts für Kompetenztransfers entscheiden. Diese Beurteilung ist jedoch an die Annahme geknüpft, dass Regierungen mit der Abgabe von Entscheidungsbefugnissen nicht vor allem eigene Präferenzen innenpolitisch durchsetzen wollen. In der Literatur wird bisher zu wenig beachtet, dass der Europäische Rat aus Regierungsvertretern besteht, die parteipo-

27 Christopher Lord/David Beetham: Legitimizing the EU: Is there a 'Post-parliamentary Basis' for its Legitimation?, in: Journal of Common Market Studies 3/2001, S. 443-462, hier S. 449.

28 John Rawls: Eine Theorie der Gerechtigkeit, Frankfurt am Main 1979, S. 44-45.

29 Joshua Cohen: Deliberation and Democratic Legitimacy, in: James Bohman/Wiliam Rehg (Hrsg.): Deliberative Democracy: Essays on Reason and Politics, Cambridge, Massachusetts/London 1997, 67-91, hier S. 73. 
litisch gebunden sind. Die Entscheidung, Kompetenzen dem nationalen Zugriff zu entziehen, war in der Vergangenheit immer wieder auch parteipolitisch motiviert. Die Unabhängigkeit der Europäischen Zentralbank erschien Mitte-rechts-Regierungen im Vorlauf zum Maastrichter Vertrag wichtig, weil sie eine unsolide Haushaltspolitik nachfolgender Regierungen fürchteten. Sie konnten auch deshalb die seit dem Den Haager Gipfel (1969) andauernde Auseinandersetzung zwischen „Ökonomisten“ und „Monetaristen“30 über den richtigen Weg zur Währungsunion für sich entscheiden, weil in zehn von zwölf Mitgliedsländern Mitte-rechts-Koalitionen regierten. Die Umsetzung der Wirtschafts- und Währungsunion entsprach im Ergebnis der Vorstellung christdemokratischer Regierungschefs und der EVPPosition. ${ }^{31}$

Da die Parlamentswahlen in den Mitgliedstaaten gemeinsame Trends aufweisen, unterliegt der Europäische Rat im Laufe der Zeit starken Links-rechts-Schwankungen. Statt einer permanenten Großen Koalition haben sich in der Vergangenheit Mitte-rechts- und Mittelinks-Mehrheiten abgewechselt - mit einem deutlichen Übergewicht vor allem christdemokratischer Parteien. ${ }^{32}$ Diese Mehrheiten nutzen Phasen der Dominanz zur Konstitutionalisierung ihrer politischen Präferenzen. ${ }^{33}$ Wenn jedoch die Delegation von Befugnissen parteipolitisch motiviert ist, kann nicht davon ausgegangen werden, dass die beauftragten Akteure Sachwalter nationaler Interessen sind. „Hoheitliche Integration“, das hatte schon Olson erkannt, ist ein probates Mittel, sich den Anliegen von „Sonderinteressengruppen“ zu entziehen. ${ }^{34}$ Moravcsik sieht das in aller Klarheit wenn er davon spricht, dass die besondere Stellung der Regierung in internationalen „Exekutivkartellen“ innerstaatliche Machtressourcen neu verteilt:

„Hier wird argumentiert, dass diese Umverteilung im allgemeinen einen Machtzuwachs für die staatliche Exekutive mit sich bringt, indem sie ihr erlaubt, die Zügel, die ihr durch Parlamente, Interessengruppen oder andere gesellschaftliche Akteure angelegt werden, zu lockern. [...] Das so umstrittene so genannte ,Demokratiedefizit‘ ist kein zufälliges Merkmal der EG, sondern ein wesentlicher Bestandteil ihres institutionellen Designs. “35

Regierungen begreifen die EU-Politik als Hebel, mit dem sie politische Ziele gegen den Widerstand innenpolitischer Interessen durchsetzen und dauerhaft verankern können. Hier zeigt sich, dass ein Spannungsverhältnis zwischen den zwei beschriebenen Legitimationskanälen besteht. Der Preis für Output-orientierte Legitimation liegt in einer verringerten Kontrolle der Regierung durch nationale Parlamente. Moravcsiks ,Liberaler Intergouvernementalismus ' enthält unausgesprochen eine Bewertung, welche Demokratieform wünschenswert ist. Durchaus theoriekonsistent sieht er eine Kongruenz zwischen der EU und dem regierungszentrierten Modell der Westminster-Demokratie. Weniger deckungsgleich ist das politische System der EU mit den gesellschaftszentrierten Demokratien Kontinental- und Nordeuropas. Falls diese Beschreibung zutrifft, ist das ,,institutionelle Design“ der EU besser mit

30 Douglas C. Kruse: Monetary Integration in Western Europe: EMU, EMS and Beyond, London u.a. 1980, S. $62-70$.

31 Karl Magnus Johansson: Another Road to Maastricht: The Christian Democrat Coalition and the Quest for European Union, in: Journal of Common Market Studies 5/2002, S. 871-893.

32 Philip Manow/Armin Schäfer/Hendrik Zorn: Europäische Sozialpolitik und Europas parteipolitisches Gravitationszentrum in den Jahren 1957-2003, in: Zeitschrift für Internationale Beziehungen 1/2006, S. 77-112.

33 Simon Hix/Christopher Lord: Political Parties in the European Union, Houndmills 1997, S. 4.

34 Mancur Olson: Aufstieg und Niedergang von Nationen. Ökonomisches Wachstum, Stagflation und soziale Starrheit, 2. Auflage, Tübingen 1991, Kapitel 5.

35 Andrew Moravcsik: Warum die Europäische Union die Exekutive stärkt: Innenpolitik und internationale Kooperation, in: Klaus-Dieter Wolf (Hrsg.): Projekt Europa im Übergang? Probleme, Modelle und Strategien des Regierens in der Europäischen Union, Baden-Baden 1997, S. 211-269, hier S. 211 und 213. 
Mehrheits- als mit Konkordanzdemokratien vereinbar, obwohl sie selbst letzteren zugerechnet wird. ${ }^{36}$ Eine Umverteilung der Einflusschancen von der Gesellschaft zur Regierung bricht aber das Neutralitätsversprechen delegierter Entscheidungen.

Der Europäische Rat repräsentiert die Präferenzen der Wähler zudem nur unvollständig. Da in ihm nur die Regierungen vertreten sind, finden die Oppositionsparteien keine Berücksichtigung. Die Zusammensetzung intergouvernementaler Gremien wirkt wie ein Filter der Wählerpräferenzen. Ideologisch extremere oder europaskeptische Parteien sind in ihnen kaum vertreten. Eine nur selektive Repräsentation wirft demokratietheoretische Probleme auf, weil die nationalen Parlamente kaum zur effektiven Kontrolle der Exekutive fähig sind. ${ }^{37}$ Grundlegende Weichenstellungen über die Integrationstiefe werden ohne ausreichende parlamentarische Kontrolle vorgenommen. Das führt zu einem Risss zwischen den Wählern und ihren Repräsentanten, der die Legitimität der Integration gefährdet: "Voters and their representatives are living in two different worlds, and the positions of those voters who are sceptical or opposed are almost totally ignored." 38

Die Repräsentationslücke in intergouvernementalen Gremien wirft ein interessantes Licht auf die These, dass Europawahlen im Kern Nebenwahlen seien, bei denen die Unzufriedenheit mit innenpolitischen Themen zum Ausdruck käme. Die Literatur zu Europawahlen besagt, dass Oppositionsparteien im Vergleich zur nationalen Wahl Stimmen hinzugewinnen, während große Regierungsparteien an Zustimmung verlieren. Besonders erfolgreich sind darüber hinaus kleine Parteien an den Rändern des politischen Spektrums, die zum Teil wie beispielsweise der Front National - vehement gegen die EU opponieren. ${ }^{39}$ Doch wenn europaskeptische Parteien gerade in den Wahlen zum Europäischen Parlament überproportional gut abschneiden liegt es nahe, dass die Wähler auch tatsächlich gegen ,zuviel Europa“ protestieren. Der Erfolg europaskeptischer Parteien muss dabei kein Hinweis auf eine nationalistische Orientierung der Wähler sein. Die These des ,overshooting“ besagt, dass Wähler Parteien unterstützen, die extremere Präferenzen als sie selbst haben, wenn sie sich dadurch eine Korrektur der Regierungspolitik erhoffen. ${ }^{40}$ Insofern können Europawahlen als Protestwahlen gedeutet werden - Gegenstand des Protests sind allerdings europapolitische Themen.

Die Bürger verfügen über zwei Einflusskanäle in der EU, doch wie diese Kanäle gegenwärtig genutzt werden weist paradoxe Züge auf. Über nationale Parlamentswahlen können die Wähler beeinflussen, wie integrationsfreundlich der Europäische Rat ist. Europaskepti-

36 Manfred G. Schmidt: Der konsoziative Staat. Hypothesen zur politischen Struktur und zum politischen Leistungsprofil der Europäischen Union, in: Edgar Grande/Markus Jachtenfuchs (Hrsg.): Wie problemlösungsfähig ist die EU? Regieren im europäischen Mehrebenensystem. Baden-Baden 2000, S. 33-58; Arend Lijphart: Patterns of Democracy. Government Forms and Performance in Thirty-six Countries, New Haven/London 1999, S. 42-47.

V. Schmidt kommt zum gegenteiligen Urteil. Aus ihrer Sicht sind Konkordanzdemokratien besser als Mehrheitsdemokratien auf die Verhandlungszwänge des EU-Systems vorbereitet. Allerdings begreift sie den Rat als ein Gremium, in dem das ,nationale Interesse' dominiert. Weitere empirische Studien müssen klären, in welchen Politikfeldern nationale, sektorale oder parteipolitische Faktoren das Verhalten der Regierung prägen. Vivien A. Schmidt: Adapting to Europe: Is it Harder for Britain?, in: British Journal of Politics and International Relations 1/2006, S. 15-33, hier S. 25.

37 Tapio Raunio: Always One Step Behind? National Legislatures and the European Union, in: Government and Opposition 2/1999, S. 180-202.

38 Stefano Bartolini: Mass Politics in Brussels: How Benign Could It Be?, in: Zeitschrift für Staats- und Europawissenschaften 1/2006, S. 28-56, hier S. 49.

39 Michael Marsh: Testing the Second-Order Election Model after Four European Elections, in: British Journal of Political Science 4/1998, S. 591-607; Hermann Schmitt/Karlheinz Reif: Der Hauptwahlzyklus und die Ergebnisse von Nebenwahlen: Konzeptuelle und empirische Rekonstruktionen am Beispiel der Europawahlen im Wahlzyklus der Bundesrepublik, in: Andreas M. Wüst (Hrsg.): Politbarometer, Opladen 2003, S. 239-254.

40 Orit Kedar: When Moderate Voters Prefer Extreme Parties: Policy Balancing in Parliamentary Elections, in: American Political Science Review 2/2005, S. 185-199. 
sche Wähler sollten daher in nationalen Wahlen ihren Widerspruch gegen Vertiefung und Erweiterung ausdrücken, denn diese Weichenstellungen werden intergouvernemental vorgenommen. Dies geschieht jedoch nicht, weil innenpolitische Themen die Wahlentscheidung bestimmen. Der zweite Einflusskanal sind die Europawahlen. Hier können die Wähler die Politikgestaltung der EU in Bereichen beeinflussen, in denen ein Kompetenztransfer bereits stattgefunden hat. Bei der Ausformulierung konkreter Policies verfügt das Europäische Parlament über mehr Macht als bei der Konstitutionalisierung der EU. Doch gerade bei Europawahlen schneiden europaskeptische Parteien besonders gut ab, die wenig Interesse an politischer Gestaltung haben. Das Zusammenspiel beider Mechanismen führt dazu, dass Wahlen wenig Einfluss auf die europäische Politik ausüben:

„The choices in both channels become increasingly irrelevant to the outputs of the system, and the behaviour and preferences of citizens constitute virtually no formal constraint on, or mandate for, the relevant policy-makers. Decisions can be taken by political elites with more or less free hand." 41

Glaubwürdigkeit: Liegt das primäre Ziel von Delegation in höherer Glaubwürdigkeit, muss der beauftragte Akteur ohnehin vom Ansinnen wechselnder Mehrheiten entkoppelt werden. Jede Erwartung an eine unabhängige Notenbank, den volatilen Präferenzen von Regierungen oder der Bevölkerung zu folgen, erscheint unangebracht, denn dies würde den Zweck der Delegation untergraben. Glaubwürdigkeit wird gerade durch die Unabhängigkeit auch von Regierungswechseln erzeugt. Mit dem Verweis auf die Gültigkeit internationaler Verträge über eine Wahlperiode hinaus begründet Majone die Notwendigkeit einer dauerhaften Bindung der Mitgliedstaaten an gemeinsame Ziele. ${ }^{42}$ Dies gewährleistet insbesondere die Übertragung von Entscheidungsbefugnissen an supranationale Akteure wie die EZB oder den EuGH. Hierbei wird, wie sonst in Public-choice-Analysen, den politischen Akteuren eine Handlungsrationalität unterstellt, die keine Möglichkeit opportunistischen Verhaltens ausschlägt. Im Gegensatz zu dieser Annahme wurde die Unabhängigkeit der Bundesbank trotz wiederkehrender Regierungswechsel nicht angetastet, obwohl dafür ein einfaches Gesetz genügt hätte. Die bloße Möglichkeit, politische Entscheidungen rückgängig zu machen, führt nicht zwangsläufig zur Umgestaltung bestehender Institutionen. Dennoch gewährt das Prinzip der Reversibilität im Nationalstaat die Legitimität delegierter Machtausübung. In der Europäischen Union ist dieses Prinzip mit solchen Hürden belastet, dass supranationale Akteure ein hohes $\mathrm{Maß}$ an Autonomie genießen, mit dem sie sich gegenüber Forderungen der Wähler weitgehend abschotten können. ${ }^{43}$

Im Nationalstaat wird der freiwillige Verzicht auf das politisch Machbare als Akzeptanz der Entscheidungen nicht-majoritärer Institutionen gewertet. Für Akteure auf der EU-Ebene kann dies nicht in gleicher Weise angenommen werden. Da einmal auf die EU übertragene Kompetenzen ihr in der Regel nur einstimmig entzogen werden können, ist es den Mitgliedstaaten selbst nach einem Umschwung in der Wählergunst nahezu unmöglich, frühere Entscheidungen rückgängig zu machen. ${ }^{44}$ Moravcsiks Argumentation, dass das Muster der Delegation in der EU sich nicht von dem in den Mitgliedstaaten unterscheidet, greift deshalb zu kurz. ${ }^{45}$ Die Verfügbarkeit der Option, Delegation rückgängig zu machen und agents das

41 Mair: Popular Democracy and the European Union Polity, S. 9.

42 Majone: Two Logics of Delegation, S. 106-107.

43 Michael Zürn: Über den Staat und die Demokratie im europäischen Mehrebenensystem, in: Politische Vierteljahresschrift 1/1996, S. 27-55, hier S. 41.

44 Paul Pierson: The Path to European Integration: A Historical Institutionalist Analysis, in: Comparative Political Studies 2/1996, S. 123-163, hier S. 142-144.

45 Moravcsik: In Defence of the 'Democratic Deficit', S. 613. 
Mandat zu entziehen, trägt zur Kontrolle und Legitimität ihrer Handlungen bei. Der Bereich technokratischer Entscheidungen kann nur großzügig bemessen werden, wenn eine effektive Kontrolle der Stellvertreter gewährleistet ist. Das ist allerdings in der Europäischen Union noch schwieriger zu erreichen als im Nationalstaat.

Keine Generalbevollmächtigung: Nachträgliche Kontrolle ist besonders wichtig bei einem ,freien Mandat' der Stellvertreter, das deren Handlungsautonomie vorab nicht eng eingrenzt. Sind supranationale Akteure nur auf ein allgemeines Ziel verpflichtet, bei dem sie ihr Mandat eigenständig ausgestalten und die Mittel zur Umsetzung frei wählen, ist die vorausschauende Kontrolle durch die Auftraggeber kaum möglich. Da die Glaubwürdigkeit mit der Autonomie der agents zusammenhängt, können ihnen die Hände in jenen Politikfeldern nicht gebunden werden, die für zeitinkonsistentes Verhalten anfällig sind. ${ }^{46}$ Dies bedeutet aber auch, dass die Reichweite der Stellvertreter-Entscheidungen nicht klar eingrenzbar ist und scheinbar technische Entscheidungen auf andere Politikbereiche ausgreifen. In der Geldpolitik ist es beispielsweise eine gewollte Folge des Kompetenztransfers auf die unabhängige Notenbank, dass ihre Entscheidungen den Spielraum in der Lohn- und Fiskalpolitik begrenzen. ${ }^{47}$ Die Glaubwürdigkeit nicht-inflationärer Politik wird nur erreicht, wenn die Zentralbank eine nicht-akkommodierende Geldpolitik verfolgt. In diesem Fall antizipieren die anderen Wirtschaftsakteure negative Konsequenzen einer restriktiven Geldpolitik und passen ihre Entscheidungen dieser Erwartung an. Mit der Verlagerung der Zinspolitik auf eine unabhängige Behörde wird nicht nur dieser Bereich dem politischen Zugriff entzogen, sondern - so die Hoffnung der Befürworter - auch die Lohn- und Fiskalpolitik diszipliniert. Als Resultat geldpolitischer Delegation werden zentrale wirtschaftspolitische Entscheidungen entpolitisiert und einer nicht-majoritären Institution untergeordnet. Auch in diesem Fall begründet der Verweis auf den Nationalstaat ein ähnliches Vorgehen der EU nicht, da in den Mitgliedstaaten Delegation auf Zeit gewährt wird und prinzipiell durch die Parlamentsmehrheit widerrufbar ist. Im Nationalstaat besteht der ,Schatten der Hierarchie“48 auch bei delegierten Kompetenzen. Die Entpolitisierung politischer Entscheidungen schwächt gerade in einem politischen System ohne institutionalisierte Opposition und ohne effektive Reversibilitäts-Androhung die Legitimität.

Die zentrale Schwierigkeit delegationstheoretischer Argumente liegt im Ausblenden politischer Konflikte: erleichterte Beschlussfassung und autonome Entscheidungen durch supranationale Akteure werden mit einer erhöhten Problemlösungsfähigkeit gleichgesetzt. Das politische System muss aus dieser Sicht befähigt werden, Entscheidungen ohne Reibungsverluste zu treffen. Vereinfachte Entscheidungsregeln oder der vollständige Souveränitätstransfer sollen die Gesamtwohlfahrt maximieren. Nicht aus Zufall greifen die Verfechter der Gegenthese auf die Literatur zur Zentralbankunabhängigkeit zurück. Stabile Preise werden überwiegend als wünschenswert angesehen, und unabhängige Notenbanken garantieren die Umsetzung dieses Ziels. ${ }^{49}$ Doch in anderen Politikbereichen besteht ein solcher Konsens nicht. Das Demokratieverständnis von Moravcsik und Majone setzt die Ziele als gegeben voraus, sieht aber deren Umsetzung durch zu viel Politik gefährdet. Dagegen lässt sich ein-

46 Finn E. Kydland/Edward C. Prescott: Rules Rather than Discretion: The Inconsistency of Optimal Plans, in: The Journal of Political Economy 3/1977, 473-492.

47 Otmar Issing: On Macroeconomic Policy Co-ordination in EMU, in: Journal of Common Market Studies 2/ 2002, 345-358, hier 346

48 Fritz W. Scharpf: Interaktionsformen. Akteurzentrierter Institutionalismus in der Politikforschung, Opladen 2000, S. 323 und S. 334-335.

49 Andreas Busch: Die politische Ökonomie der Inflation, in: Herbert Obinger/Uwe Wagschal/Bernhard Kittel (Hrsg.): Politische Ökonomie. Demokratie und wirtschaftliche Leistungsfähigkeit, Opladen 2002, S. 175-197, hier S. 184-186. 
wenden, dass Mehrheitsabstimmungen und Delegation nicht die Legitimität politischer Entscheidungen erhöhen, sondern die politische Repräsentation aushöhlen, wenn Interessengegensätze bestehen und politische Maßnahmen umstritten sind. Falls das Ergebnis von der unterlegenen Minderheit nicht als auch für sie vorteilhaft anerkannt wird, hat der Output keine legitimierende Kraft.

Die Auseinandersetzung mit zwei prominenten Vertretern der Gegenthese zum EU-Demokratiedefizit hat gezeigt, dass die analytische Trennung von Output- und Input-orientierter Legitimation sehr voraussetzungsreich ist. Sie benötigt einen Maßstab, mit dem sich , gutes Regieren ' bemessen lässt. Dies mag in einigen Politikfeldern möglich sein. Doch häufig ist umstritten, was die richtige Politik ist, und selbst technische Entscheidungen können Verteilungskämpfe nach sich ziehen. Welche Politik angemessen erscheint, kann nicht ex cathedra vom Theoretiker, sondern muss im demokratischen Verfahren entschieden werden. Falls wir keine privilegierte Beobachterposition in Anspruch nehmen, sind der offen ausgetragene Streit um Alternativen und die Möglichkeit für die Wähler notwendig, zwischen verschiedenen Angeboten wählen zu können. Output-Legitimation hängt zwingend davon ab, dass vorab demokratisch entschieden wird, welche Politik verfolgt werden soll. Insofern wird hier für den Vorrang Input-orientierter Legitimation argumentiert.

Die Kritiker der Demokratiedefizitthese umgehen die Schwierigkeit unterschiedlicher Präferenzen, indem sie für manche Entscheidungsarten - z.B. Regulierung - in Anspruch nehmen, dass diese im Interesse aller seien und daher dem politischen Streit entzogen werden könnten. Da der Zweck als gegeben angenommen wird, müssen effektive Mittel zur Verwirklichung gefunden werden. Der Rückgriff auf delegationstheoretische Argumente ist besonders geeignet, weil Delegation ein Auftragsverhältnis beschreibt, bei dem nachgeordnete Behörden bereits getroffene Entscheidungen umsetzen. Die ausführenden Akteure gleichen in diesem Verständnis einer Bürokratie, die auf die effektive und effiziente Umsetzung politischer Beschlüsse verpflichtet ist. Input-orientierte Legitimation ist unumgänglich, wenn gefragt wird, warum bestimmte Ziele und nicht andere verfolgt werden, falls es unterschiedliche Wege zum Ziel gibt oder falls es nur erreicht werden kann, wenn einem Teil der Bevölkerung ein Opfer abverlangt wird. Sind diese Aspekte strittig, ist die Auseinandersetzung um Alternativen notwendig. Für die Legitimität der Politik ist in diesem Fall entscheidend, ob die Repräsentation unterschiedlicher Interessen gewährleistet wird. ${ }^{50}$

\section{Schleichende Aushöhlung nationaler Politik}

Mit Grande lassen sich zwei Varianten der Kritik am EU-Demokratiedefizit benennen: ${ }^{51}$ Die ,Standardkritik“ beruft sich auf die schwache Rolle des Europäischen Parlaments in der europäischen Gesetzgebung. Dieses institutionelle Defizit ließe sich durch eine weitere Aufwertung des Europäischen Parlaments beheben. Tiefer geht die ,substanzielle“ Variante der Kritik, die besagt, dass Demokratie ohne Demos nicht möglich und die EU schlicht nicht demokratiefähig ist. ${ }^{52}$ Die Gegenthese - das macht einen Teil ihrer Attraktivität aus - erklärt diese Unterscheidung letztlich für irrelevant, da die Entscheidungen der EU-Institutionen nicht weiter demokratisiert werden müssen, erfüllen sie doch jene Gemeinwohl fördernden Aufgaben, die auch im Nationalstaat von nicht-majoritären Institutionen übernommen wer-

50 Thomas Christiano: The Significance of Public Deliberation, in: James Bohman/William Rehg (Hrsg.): Deliberative Democracy: Essays on Reason and Politics, Cambridge, Massachusetts/London 1997, S. 243-277, hier 258.

51 Edgar Grande: Demokratische Legitimation und europäische Integration, in: Leviathan 3/1996, S. 339-360.

52 Peter Graf Kielmansegg: Integration und Demokratie, in: Markus Jachtenfuchs/Beate Kohler-Koch (Hrsg.): Europäische Integration, Opladen 2003, S. 49-83, hier S. 57-61. 
den. Dieser Aufsatz wendet sich gegen diese Auffassung, da die Vollmachten der Europäischen Union in vielen Politikfeldern über regulative Politik hinausgehen. Die Entscheidungen der Europäischen Union beschränken sich nicht ausschließlich auf ParetoVerbesserungen, sondern produzieren Gewinner und Verlierer. Das Demokratiedefizit besteht darin, dass die Bedeutung der EU für die nationale Politik zunimmt, ohne dass mit diesem Zuwachs eine Politisierung der Entscheidungen einhergeht. Im Gegenteil, besonders einflussreiche Institutionen, wie zum Beispiel die EZB, werden dauerhaft dem demokratischen Zugriff entzogen - und das in einem höheren Maß als es im Nationalstaat der Fall war.

Die Überführung umkämpfter Politikfelder in die Vergemeinschaftung war in der Vergangenheit die raison d'être der Integration. Politik sollte entideologisiert, rationalisiert und bürokratisiert werden, um die Problemlösungskapazität bei verminderter Konflikthaftigkeit zu erhöhen. Die politische (und integrationstheoretische) Auseinandersetzung wurde um die Frage geführt, welche Bereiche der gemeinschaftlichen Aufsicht unterstellt werden sollten. Je mehr sich jedoch die Auseinandersetzung von der ,vertikalen“ auf die ,horizontale“ Links-rechts-Achse verschiebt, je mehr der politische Inhalt und nicht die Regelungsebene kontrovers ist, desto nötiger ist die öffentliche Diskussion über die richtige Politik. ${ }^{53}$ Die Delegationsdebatte bewegt sich weit gehend in der vertikalen Welt und bestreitet die horizontale Auseinandersetzung zwischen konkurrierenden "Integrationsprojekten“. 54 Doch wenn EU-Entscheidungen transformativ in nationale Wohlfahrtsstaaten und Spielarten des Kapitalismus eingreifen, benötigen sie mehr als indirekte Legitimation. Die schleichende Aushöhlung nationaler Politik führt sonst dazu, dass aus dem europäischen ein nationales Demokratiedefizit wird.

53 Philipp Genschel: Markt und Staat in Europa, in: Politische Vierteljahresschrift 1/1998, S. 55-79.

54 Liesbet Hooghe/Gary Marks: The Making of a Polity: The Struggle over European Integration, in: Herbert Kitschelt u.a. (Hrsg.): Continuity and Change in Contemporary Capitalism, Cambridge 1999, S. 70-97. 